\title{
Identifying mechanisms that predict weight trajectory after bariatric surgery: rationale and design of the biobehavioral trial
}

\author{
Leslie J. Heinberg, Ph.D. ${ }^{\mathrm{a}, *}$, Dale S. Bond, Ph.D. ${ }^{\mathrm{b}}$, Ian Carroll, Ph.D. ${ }^{\mathrm{c}}$, Ross Crosby, Ph.D. ${ }^{\mathrm{d}, \mathrm{e}}$, \\ Anthony Fodor, Ph.D. ${ }^{\mathrm{f}}$, Farnaz Fouladi, Ph.D. ${ }^{\mathrm{f}}$, John Gunstad, Ph.D. ${ }^{\mathrm{g}}$, \\ James Mitchell, M.D. ${ }^{\text {d,e }}$, Christine Peat, Ph.D. ${ }^{\text {c }}$, Kristine Steffen, PharmD, Ph.D. ${ }^{\text {h,i }}$ \\ ${ }^{a}$ Cleveland Clinic Lerner College of Medicine of Case Western Reserve University, Cleveland, Ohio \\ ${ }^{b}$ Weight Control and Diabetes Research Center, The Miriam Hospital/Brown Alpert Medical School, Providence, Rhode Island \\ ${ }^{c}$ University of North Carolina School of Medicine, Chapel Hill, North Carolina \\ ${ }^{d}$ Department of Psychiatry and Behavioral Science, Sanford Center for Biobehavioral Research, Fargo, North Dakota \\ ${ }^{e}$ University of North Dakota School of Medicine and Health Sciences, Fargo, North Dakota \\ ${ }^{f}$ University of North Carolina School of Medicine at Charlotte, Charlotte, North Carolina \\ ${ }^{g}$ Department of Psychological Science and Brain Health Research Institute, Kent State University, Kent, Ohio \\ ${ }^{h}$ North Dakota State University, Fargo, North Dakota \\ ${ }^{i}$ University of North Dakota School of Medicine and Health Sciences, Fargo, North Dakota
}

Received 31 January 2020; accepted 11 June 2020

\begin{abstract}
Bariatric surgery is currently the most efficacious and durable intervention for severe obesity. The most commonly performed procedures in the United States are the Roux-en-Y gastric bypass and the sleeve gastrectomy, which involve significant anatomic and physiologic alterations that lead to changes in behavior and biology. Unfortunately, many patients experience suboptimal weight loss and/or substantial weight regain. Eating and physical activity/sedentary behaviors, mood, cognition, and the gut microbiome all change postoperatively and have an association with weight change. The longitudinal relationship between changes in the gut microbiome and postoperative weight trajectory has not been explored thoroughly, and the interactive associations among the gut microbiome and the other variables that impact weight have been similarly understudied.

The following is a methods and design description for a prospective, 24-month longitudinal study of 144 bariatric surgery patients, at 2 sites, that aimed to identify predictors of weight loss trajectories over 24 months after Roux-en-Y gastric bypass and the sleeve gastrectomy. Specifically, the study will examine the relationships between empirically supported behavioral and biological variables and their combined impact on postoperative weight trajectories. Novel data collection will include intensive measurement of problematic eating behaviors and diet and physical activity postoperatively, which may be altered in parallel with, or in response to, changes observed in the gut microbiota. Identifying postoperative predictors of weight loss and co-morbidity resolution should inform development of novel interventions that are tailored to individual patients' risk profiles to optimize and sustain more favorable weight trajectories. (Surg Obes Relat Dis 2020;16:1816-1826.) (C) 2020 American Society for Bariatric Surgery. Published by Elsevier Inc. All rights reserved.
\end{abstract}

Key words:

Bariatric surgery; Weight loss; Microbiome; Gut-brain axis; Eating behavior; Physical activity

Supported by National Institutes of Health Grant R01 DK112585-01.

*Correspondence: Leslie J. Heinberg, Ph.D., Cleveland Clinic Lerner College of Medicine, 9500 Euclid Avenue/M61, Cleveland, OH 44195.
E-mail address: heinbel@ccf.org (L.J. Heinberg). 
The most effective treatment for severe obesity is bariatric surgery [1], with the Roux-en-Y gastric bypass (RYGB) and the sleeve gastrectomy (SG) being the most commonly performed procedures in the United States [2]. However, the multisite Longitudinal Assessment of Bariatric Surgery study found that a significant subset of patients $(\sim 25 \%)$, do not achieve expected weight loss outcomes [3]. Reviews also suggest that most patients regain some weight with approximately $20 \%$ regaining the majority of lost weight [4]. These suboptimal outcomes can result in lack of improvement or recurrence of medical co-morbidity $[5,6]$.

Prior research has shown significant associations between weight loss after bariatric surgery and variables involving eating pathology, such as "loss of control" eating [5,7-9], "grazing," eating when not hungry [5,10,11], nocturnal eating, and stress-induced eating [5,10-12]. Residual depressive symptoms also have been shown to negatively impact weight outcomes [13] as has lower physical activity and higher sedentary behavior [14-16]. Differences in executive function may impact eating, adherence, and physical activity [17].

Beyond behavioral variables, biological factors-namely the gut microbiome - have been a recent area of focus in the context of bariatric surgery. Human and animal work have established obesity-associated permutations in the gut microbiota with some studies demonstrating causal relationships between specific changes in the gut microbiota and weight loss [18]. Numerous anatomic and physiologic changes follow RYGB and SG that may contribute to changes in the microbial flora of the distal gut. Prospective research show increases in specific phyla, such as Proteobacteria, after RYGB that consistently correlate with changes in weight, serum leptin, and variably with inflammation markers including C-reactive protein [19]. The ratio of Firmicutes to Bacteroidetes has been shown to decrease after RYGB after controlling for sex, age, and medication [19]. Of note, past studies have not adequately controlled for dietary intake. This is considered to be a significant deficiency of the extant literature [20]. The collective body of evidence suggests there is bidirectional communication between the gut microbiome and brain function (i.e., the gut-brain axis) [21], which may impact behavior, but this interface has not been well studied in bariatric samples.

\section{Aims of the BioBehavioral Trial}

The contributors to suboptimal weight loss or regain after bariatric surgery are poorly understood and likely involve complex interactions between behavioral and biological variables. Our first aim was to describe the prospective relationships among weight change and variations in postoperative problematic eating behaviors, physical activity/sedentary behaviors, mood, and cognitive function, and whether these relationships are moderated by surgical procedure. We hypothesize that patients who report fewer problematic eating behaviors, greater levels of physical activity/lower levels of sedentary behavior, less depressive symptoms, and better cognitive function, will experience a more favorable weight loss trajectory and greater percent excess weight loss (\%EWL) at 24 months after surgery. Furthermore, we hypothesize that these relationships will be stronger for participants receiving RYGB versus SG. Aim 2 characterized the prospective relationships between postoperative weight trajectory and changes in the composition of the gut microbiome and further explore whether this relationship is moderated by surgical procedure. We hypothesized that the presence and abundance of specific taxa within the intestinal microbiota will be demonstrated in patients who experience the most favorable weight outcomes and greatest 24-month \%EWL, and that these relationships will be moderated by surgical procedure. A final exploratory aim assessed whether the microbiome gut-brain axis is a mechanistic determinant of weight trajectory after bariatric surgery. We hypothesized that changes in the composition of the gut microbiome would mediate the relationship between weight trajectory after RYGB or SG and behavioral variables of interest. This aim, within a longitudinal study, allowed us to begin to better understand the interaction between biological and behavioral variables on explaining weight loss trajectories.

\section{Methods}

This study was designed as a prospective, longitudinal assessment of behavioral, psychological, and biological factors temporally related to weight loss trajectory over 2 years after RYGB or SG. It was submitted to NIDDK in response to RFA-DK-16-017 (Psychosocial and Behavioral Mechanisms in Bariatric Surgery). Data collection occurs at two sites (Sanford Center for Biobehavioral Research [CBR], in collaboration with North Dakota State University, in Fargo, ND; and Cleveland Clinic Bariatric and Metabolic Institute, Cleveland, OH. Inability to engage in physical activity or dietary monitoring Self-report Recruitment takes place through the Sanford Eating and Weight Management Center through an established collaborative relationship with the Neuropsychiatric Research Institute in Fargo, ND, and through the Cleveland Clinic Bariatric and Metabolic Institute, Cleveland, $\mathrm{OH}$.

\section{Participants and recruitment}

Participants who are in the evaluation process for RYGB and SG are offered the opportunity to volunteer for this study. A total of 144 participants undergoing bariatric surgery ( $\sim 50 \%$ RYGB and 50\% SG) will be enrolled in this study. In anticipation of a rate of attrition of approximately $30 \%$, this enrolled sample is expected to yield a final sample size of 100, which will provide adequate power to address 
the stated hypotheses. Enrolled participants are assessed at baseline (presurgery) and specified time points $(1,6,12$, 18, 24 mo) after surgery (Fig. 1).

Recruitment takes place through REDACTED FOR BLIND REVIEW in REDACTED FOR BLIND REVIEW, and through the REDACTED FOR BLIND REVIEW. Criteria for enrollment were carefully considered and chosen to balance generalizability with the need to reduce some confounding variables (Table 1). Additional data that could influence the microbiome are collected and will be used as covariates in analyses if appropriate (e.g., comorbid medical conditions, medication use, surgical history [especially of cholecystectomy/appendectomy], nutritional supplement use, etc.).

\section{Study assessments and timeline}

\section{Screening visit}

Participants who provide informed consent at a presurgical evaluation visit in the bariatric clinic are screened for study eligibility by research staff according to the list of inclusion and exclusion criteria (Table 1). All criteria are in place to protect participant safety and to avoid introducing unnecessary confounding variables into the study data. At screening, each participant completes a medical history to assess for inclusion/exclusion criteria. Participants also have the first Structured Clinical Interview (SCID-5) [22] administered during the screening visit to assess for psychopathology that may affect protocol compliance. Participants who meet all eligibility crtieria are invited to enroll in the study and complete study assessements according to the schedule described below. Participants are required to complete study baseline assessments before beginning any prescribed presurgical diet.

Study visits (presurgery and 1, 6, 12, 18, and 24 mo postsurgery)

With the exception of the stuctured clinical interview for psychological disorders, all assessments are completed at each study visit (Fig. 1). The following assessments are completed:

\section{Measures and variables of interest}

Weight, \%EWL, body mass index

Height and weight data are collected at each study visit. Participants are weighed in light clothing without shoes. Participants' ideal weight is calculated based upon a body mass index $(\mathrm{BMI})=25$ at baseline to facilitate determination of \%EWL after surgery. To facilitate comparison between studies, data are recorded in weight (lbs, $\mathrm{kg}$ ), body BMI units $\left(\mathrm{kg} / \mathrm{m}^{2}\right)$, and \%EWL.

\section{Dietary recall: the ASA24 automated self-administered 24-hour}

The ASA24 dietary recall was developed by the National Cancer Institute. The ASA24 has been validated for research use and offers a web-based platform for the collection of automated, self-administered, 24-hour dietary recalls

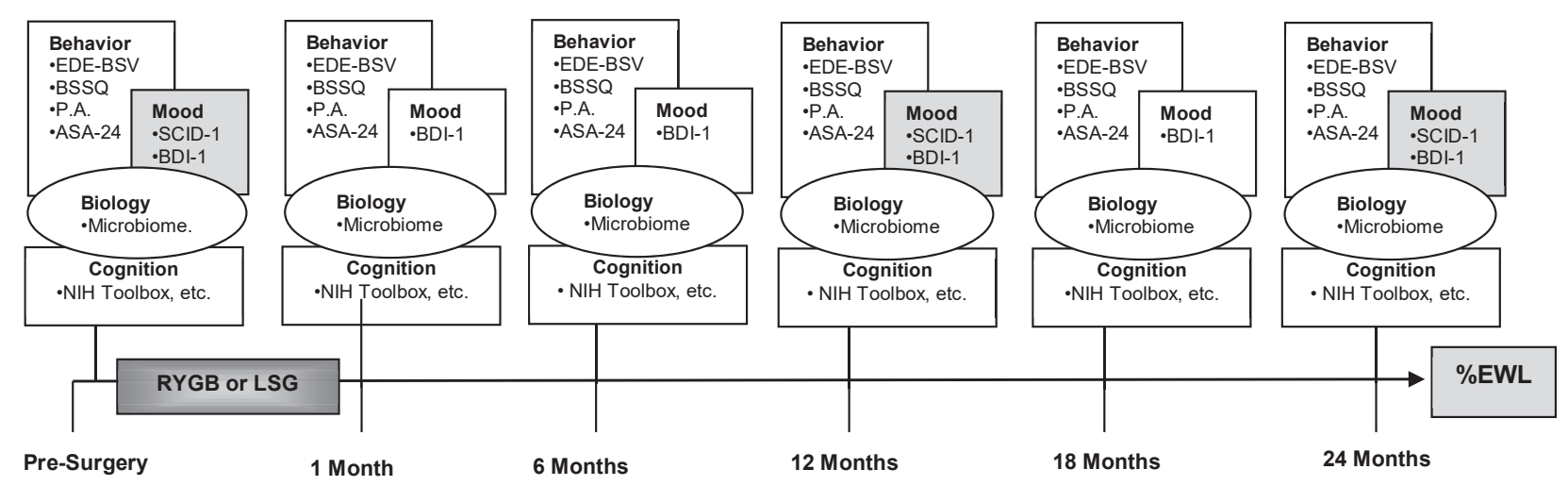

EDE-BSV=Eating Disorders Examination-Bariatric Surgery Version

$\mathrm{BSSQ}=$ Bariatric Surgery Self-Management Questionnaire

$\mathrm{PA}=$ Physical Activity

ASA-24=The ASA24 Automated Self-administered 24-Hour

SCID-1=Structured Clinical Interview for DSM-5)

BDI-1=The Beck Depression Inventory 1

Fig. 1. Schedule of study assessments. 
Table 1

Study enrollment criteria

Inclusion criteria

Male or female between the ages of 18 and $65 \mathrm{yr}$, inclusive

In evaluation for Roux-en-Y gastric bypass or sleeve gastrectomy as primary procedure

Planning to be available for $2 \mathrm{yr}$ of follow-up after surgery

Exclusion criteria

Alcohol or substance use disorder in the past year

Severe psychiatric disorder that may affect the ability of the participant's ability to comply with the protocol

Tobacco use in the prior year

Current medication known to significantly influence gastrointestinal transit time (e.g., opioids, glucagon-like peptide-1 agonists, metoclopramide) or is being taken routinely for weight loss (e.g., phentermine, topiramate)

Use of any oral or injectable antibiotic in the past month

Use of commercially available prebiotic or probiotic in the past month

History of significant intestinal disease or disorder that would influence the microbiome (e.g., Crohn's disease, etc.)

History of significant gastrointestinal surgery that would influence the microbiome, not including cholecystectomy or appendectomy

Inability to engage in physical activity or dietary monitoring or any medical condition that would put the participant at risk in the study

Positive urine drug screen for a nonprescribed medication

Pregnancy or breast feeding

Method of assessment

Photo identification

Surgery department

Self-report

Method of assessment

Structured interview

Structured interview

Self-report

Medical history

Medical history

Medical history

Medical history

Medical history

Self-report

Urine drug screen

Self-report (food, fluids, vitamins, and supplements). The ASA24 is based upon the U.S. Department of Agriculture Automated Multiple-Pass Method, which is validated and provides accurate estimations of mean total energy and protein consumption in biomarker testing [23,24]. The system has been used widely in nutrient research and is considered the gold standard method of assessment of food intake. Participants completed 3 days of dietary recall at each study visit.

\section{Psychological assessment}

The Structured Clinical Interview for DSM-5 [22] is a structured interview that is used as a diagnostic tool for psychiatric disorders following DSM-5 criteria. Psychological assessors on this protocol have regular meetings to ensure high interrater reliability on administration of the SCID. The Beck Depression Inventory [25] is a 21-item selfreport measure that assesses current depression level and symptoms of depression. It is a widely used and established measure with demonstrated reliability and validity [26]. Higher scores on the Beck Depression Inventory reflect higher levels of depression and, more broadly, negative affect [27]. There is a detailed protocol and safety plan for any acute psychiatric symptoms or suicidality.

\section{Problematic eating behaviors and adherence to recommendations}

The Eating Disorders Examination-Bariatric Surgery Version (EDE-BSV) [7] is a clinical interview measure adapted from the EDE interview [28] for use in bariatric surgery samples. The
EDE is considered to have the best validity and reliability in assessing eating disordered behavior and the EDE-BSV contains additional items that are specific to the concerns of weight loss surgery patients (e.g., dumping, plugging, etc.). Similar to the SCID, assessors meet regularly to ensure high interrater reliability. For the Bariatric Surgery Self-Management Questionnaire [29], participants complete the questionnaire, a 33-item questionnaire regarding adherence to prescribed behaviors specific to bariatric surgery. This measure asks about adherence over the previous week in eating behaviors, physical activity, dumping syndrome management, intake of fluids, supplements, fruits/vegetables/whole grains, and protein. Subscales for each domain, as well as a total score, are converted to a 0 to 99 range. Higher scores indicate better adherence. Internal consistency of the Bariatric Surgery Self-Management Questionnaire in bariatric samples is $\alpha=.83$ [29].

\section{Physical activity—objective measurement of physical activity}

Participants' daily time spent in physical activity and sedentary behavior (SB) at the different pre- and postoperative assessment periods is measured using the newest generation Actigraph monitor (i.e., ActiGraph GT9 X Link [AG]; Actigraph, LLC, Pensacola, FL, USA). The AG device is worn on the waist and combines a rigorously validated triaxial accelerometer with several advanced new features, including a wear time sensor (to detect if the device has been removed) and a secondary accelerometer, gyroscope, and magnetometer that together provide highly advanced 
data on movement, rotation, and body position (note: the AG's liquid crystal display window display for providing real-time participant feedback will be deactivated during assessments). The AG is shown to reliably measure physical activity and SB in adults over 7 days in free-living conditions [30]. Data from the AG are processed using ActiLife software version 6.11.3 (ActiGraph, LLC). Participants are asked to wear the AG on the waist during all waking hours, exclusive of bathing and swimming, for 7 consecutive days at each assessment period [31]. Participants are required to provide $\geq 4$ days of data (including 1 weekend day), defined as $\geq 600$ minutes of wear time during the hours of $7 \mathrm{AM}$ to $11 \mathrm{PM}$, to be included in analyses. Variables of primary interest will include (1) total and bout-related moderate-tovigorous physical activity, and (2) percent of daily time spent in SB overall and that accumulated in bouts (e.g., $\geq 30 \mathrm{~min}$ ) to capture the prolonged nature of $\mathrm{SB}$ and related metabolic health risks $[32,33]$.

\section{Cognitive assessment}

The National Institutes of Health Toolbox for the Assessment of Neurologic and Behavioral Function [34] was developed to assess (via a computer platform) cognitive function across the lifespan and promote generalizability of study findings across researchers. Specific measures used are consistent with those found in our past work to predict weight loss outcomes in bariatric surgery patients $[35,36]$ and include the following assessments.

\section{Attention/executive function}

The Dimension Change Card Sort Test is similar to the Wisconsin Card Sort Task and assesses hypothesis testing and ability to change mental set in the face of new contingencies. The Flanker Inhibitory Control and Attention Test is similar to the Erikson flanker task and require participants make rapid decisions about the direction of target stimuli. The List Sorting Working Memory Test requires attending to a string of visual and auditory information and reciting them back in predetermined order.

\section{Memory}

The Picture Sequence Memory Test asks individuals to learn and remember pictured objects and their actions on the screen.

\section{Processing speed}

The Pattern Comparison Processing Speed Test asks participants to quickly identify if the 2 visual patterns are the same or different.

\section{Language}

The Picture Vocabulary Test is an adaptive measure of receptive vocabulary and asks individuals to match words to target pictures among a collection of foils.

\section{Gut microbiome assessment}

\section{Fecal collection}

Fecal material is collected by each individual enrolled in our study. At the commencement of our study, and at each study visit, participants are provided a fecal sample collection kit and study cooler. For each study visit participants have the option to provide a fecal sample at the time of the study visit or may do so at home if desired. If home sample collection is preferred, participants are asked to bring the sample to the research facility, on ice/ice packs, within 24 hours of collection. Samples are labeled and stored at $-80^{\circ} \mathrm{C}$ until analysis. Each sample is transported to the molecular microbiology laboratory on dry ice where it is mechanically homogenized using a sterile spatula and aliquoted into as many 2-mL cryo-tubes as can be filled. The tubes are then labeled and immediately stored in a $-80^{\circ} \mathrm{C}$ freezer. The same procedures are used to obtain subsequent longitudinal samples from individuals $1,6,12,18$, and 24 months postsurgery.

\section{Isolation of microbial DNA}

Microbial DNA are isolated from intestinal tissue and fecal material using a phenol/chloroform extraction method combined with physical disruption of bacterial cells and a DNA clean-up kit (Qiagen DNeasy Blood and Tissue extraction kit; Qiagen, Valencia, CA, USA) as previously described [37]. The composition of the microbiome will be assessed through $16 \mathrm{~S}$ high-throughput sequencing as well as through a more comprehensive metagenomic shotgun sequencing approach.

\section{High-throughput sequencing of $16 \mathrm{~S}$ rRNA genes}

Bacterial community composition in isolated DNA from intestinal tissue and fecal material are characterized by amplification of the V6 variable region of the $16 \mathrm{~S}$ rRNA gene by polymerase chain reaction (forward: 5'-CAACGCGARGAACCTTACC-3'; reverse: 5'-CAACACGAGCTGACGAC-3') and sequencing both 5' and 3' ends using the Illumina HiSeq 2500 platform at the high-throughput sequencing core at University of North Carolina at Chapel Hill as previously described [38].

\section{Analysis of $16 S r R N A$ sequences using the DADA2 Pipeline}

$16 \mathrm{~S}$ rRNA sequence data generated by the Illumina HiSeq 2500 platform will be processed by the Divisive Amplicon Denoising Algorithm 2 (DADA2) pipeline [39], which identifies sequence variants at a $100 \%$ identity threshold. Sequence variants will be assigned to a taxonomy using a DADA2formatted reference database (silva_nr_v128_train_set.fa.gz). Read counts at each taxonomic level will be normalized using a formula as previously described to account for different sequencing depths across samples [40]. Shannon diversity index, a measurement of within-sample diversity ( $\alpha$-diversity), will be calculated using the "diversity" function from the vegan package in R (The R Project for Statistical Computing, Vienna, 
Austria). Principal coordinate analysis (PCoA) will be used to visualize between-sample diversity ( $\beta$-diversity) in the microbial composition. PCoA will be performed using Bray-Curtis dissimilarity indices and the "capscale" function from the vegan package in $R$.

\section{Analysis of $16 S$ rRNA sequences using the quantitative insights into microbial ecology pipeline}

$16 \mathrm{~S}$ rRNA sequence data generated by the Illumina HiSeq 2500 platform will be processed by the quantitative insights into microbial ecology pipeline as previously described [41]. Sequences will be clustered into operational taxonomic units based on a $97 \%$ sequence similarity (similar to species level) using UCLUST $[42,43]$. Operational taxonomic units will be assigned to a taxonomy using the Ribosomal Database Project Naive Bayes classifier [44]. The percentages of specific bacterial taxa in each sample will be summarized at 6 levels (phylum, class, order, family, genus, and species). Microbial richness measures will be generated by rarefaction of $16 \mathrm{~S}$ rRNA sequences and expressed as (1) the number of observed operational taxonomic units in each sample, and (2) the Shannon index of diversity. PCoA for each sample using unweighted and weighted UniFrac distances [45] will also be generated. PCoAs define the similarities or dissimilarities of variables that best represent the pair-wise distances between samples.

\section{Metagenomic shotgun sequencing}

We will use the Illumina HiSeq 4000 platform at the REDACTED FOR BLIND REVIEW high throughput sequencing facility to complete whole-genome shot-gun sequencing. This approach will be used to characterize the microbiome of all participants at each time point of data collection. Metagenomic data analysis will occur in collaboration with the University of North Carolina Charlotte. Metagenomic DNA sequences will be decontaminated from human genomes using KneadData (http://huttenhower.sph. harvard.edu/kneaddata). The decontaminated sequences will be taxonomically classified using the Kraken2 [46] through an automated pipeline BiolockJ (https://github. $\mathrm{com} / \mathrm{msioda} /$ BioLockJ). Microbial read counts at each taxonomic level will be normalized as described for the $16 \mathrm{~S}$ rRNA sequencing data. Microbial gene families and gene pathways will be characterized using the pipeline HUManN2 and abundances will be normalized using the HUMAnN2 tool renorm table [47]. Similar to the $16 \mathrm{~S}$ rRNA sequencing data, $\alpha$ - and $\beta$ - diversity will be calculated by Shannon Diversity Index and Bray-Curtis dissimilarity, respectively. PCoA will be used to visualize dissimilarities between samples using the "capscale" function in R.

\section{Collection of additional information that may influence the microbiome}

At study visits, additional data are recorded, which may influence the microbiome, including the use of all medications, gastrointestinal surgery history, and menstrual cycle status.

\section{Ethics and informed consent}

The study protocol was reviewed and approved by the institutional review board at both sites. Written informed consent from the patient is obtained before beginning the initial study visit. The current protocol has been registered on the National Institutes of Health's clinicaltrials.gov website.

\section{Statistical analyses and sample size estimation}

\section{Preliminary analyses}

Descriptive statistics and frequency distributions will be examined for all screening data, as well as key measures obtained at each assessment. Descriptive statistics will be compiled for the overall sample, as well as separately by site. Distribution diagnostics and outlier analyses will be performed on key variables to inform decisions about the transformation of measures for subsequent analyses. Proportional hazards regression analyses [48] will be used to evaluate whether baseline demographic characteristics or clinical characteristics are associated with study retention. Missing value analyses will be performed to identify missing data patterns and evaluate possible mechanisms for missing data (i.e., missing completely at random, missing at random, missing not at random). Variables that are associated with early dropout or missing data will be considered for use as covariates in subsequent analyses to minimize potential biases associated with missing data. Analyses will be performed to compare key baseline and clinical characteristics by site and surgical procedure. Significant differences between groups will help to inform the specifics of the analyses below as well as the use of covariates.

\section{Hypothesis testing}

\section{Hypothesis 1 a}

Patients who report fewer problematic eating behaviors, greater levels of physical activity, less mood symptoms, and better cognitive function, will experience a more favorable weight loss trajectory longitudinally after RYGB or SG and greater \%EWL from preoperative baseline to 24-months postoperatively.

Linear mixed-effects time-lagged models with random intercepts and slopes will be used to evaluate the prospective predictors of weight trajectory. The longitudinal portion of the model will be constructed following the guidelines of Singer and Willet [49], where alternative methods for modeling time (e.g., unconditional means model, unconditional linear growth model, and various unconditional nonlinear models) will be compared using the Akaike Information Criterion. Predictors will then be added to the model as time-varying effects and will be time-lagged such that predictors at time 1 will be used to predict weight at time 2, controlling for time 1 weight. Models will include both 
the main effects for predictors, as well as the predictor $X$ time interaction(s). Preliminary models will evaluate predictors separately. A multivariate model will then be constructed using significant univariate predictors.

\section{Hypothesis $1 b$}

Surgical procedure (RYGB versus SG) will moderate the relationship between eating behavior, physical activity, mood, cognitive function, and weight outcome such that these relationships will be stronger for participants receiving RYGB compared with those receiving SG.

The moderating effects of surgical procedure will be evaluated by adding a main effect for surgical procedure, as well as procedure $X$ predictor and procedure $X$ predictor $X$ time interaction(s) to the models above.

\section{Hypothesis $2 a$}

The presence and abundance of specific taxa within the intestinal microbiota will be associated with patients who experience the most favorable weight outcomes and greatest 24-month \%EWL postsurgery.

The prospective association between intestinal microbiome predictors and weight trajectories will be evaluated using linear mixed-effects time-lagged models comparable to those described for Hypothesis 1.

\section{Hypothesis $2 b$}

Surgical procedure (RYGB versus SG) will moderate the relationship between the composition of the intestinal microbiome and postsurgical weight outcomes wherein RYGB will more strongly influence the relationship between the microbiome and weight outcome compared with SG.

The moderating effects of surgical procedure will be evaluated by adding a main effect for surgical procedure, as well as procedure $X$ predictor and procedure $X$ predictor $X$ time interaction(s) to the models above.

\section{Hypothesis 3}

Changes in the composition of the gut microbiome will mediate the relationship between weight trajectory after RYGB or SG and eating and physical activity behaviors, mood, and cognitive function.

Multilevel structural equation modeling [50] with random slopes will be used to evaluate the mediating effects of changes in the gut microbiome. A 1-1-1 MSEM model (referring to the measurement level of the independent, mediator, and dependent variable, respectively) will be constructed with predictors at time 1 as the independent variable, changes in gut microbiome from time 1 to time 2 as the mediator, weight at time 2 as the dependent variable, and weight at time 1 as a covariate. This model provides nonconflated estimates of both the between- and withinpatients indirect effects.

For exploratory analyses, linear mixed-effects timelagged models with random intercepts and slopes will be used to evaluate whether weight trajectories differ as a function of baseline age, sex, surgergy type (RYGB versus SG), or the presence or absence of type 2 diabetes.

Power analysis for Hypotheses 1a and 2a were based upon multilevel Monte Carlo simulations using Mplus version 6 software [51]. Simulations assumed an unconditional linear model, 6 assessment points, a 30\% attrition rate over the 24month period, and a 2-tailed alpha of .05. The magnitude of the predictor main effect was then varied until the null hypothesis of no indirect effect was rejected on $80 \%$ of the 10,000 replications (i.e., .80 power). The proposed enrolled sample size of $n=144$ (with $\sim 100$ completing the study) provides a statistical power of .80 to detect a standardized predictor effect of .21. Sample size estimates for Hypotheses $1 \mathrm{~b}$ and $2 \mathrm{~b}$ were obtained by adding a predictor $\times$ surgery procedure interaction to the simulation models described above and then systematically varying the magnitude of the interaction effect until a power of .80 was obtained. The proposed sample size of 144 provides a statistical power of .80 to detect a standardized interaction effect of .34. Power analyses for Hypothesis 3 were based upon the methods described by Fritz and MacKinnon for determining a required sample size to detect a mediated effect [52]. Using the bias-corrected bootstrap method, a sample of size of 71 would provide adequate power (.80) to detect medium effects (.39) for both the a path (IV to mediator) and the $\mathrm{b}$ path (mediator to DV). Thus, the proposed enrolled sample of 144 ( $\sim 100$ study completers) provides sufficient power to evaluate the mechanistic role of the gut-brain microbiome.

\section{Trial status}

The trial was successfully registered on clinical trials.gov on February 27, 2017 and the institutional review board approved the study on February 9, 2017 (Cleveland) and 12/19/2016 (Fargo). Recruitment began on June 20, 17 in Cleveland and June 1, 2017 in Fargo. Seventy-nine participants at Cleveland and 70 participants at Fargo have been enrolled (over enrollment is because of participants having baseline visits but not proceeding to surgery). The final 24-month assessments for all participants are anticipated to occur by the spring of 2022 .

\section{Discussion}

Although bariatric surgery is the most effective and durable treatment for severe obesity, outcomes are variable and many experience weight regain. Research has yet to establish mechanisms for weight regain although a number of behavioral and biological variables have been posited. The study's overarching goal is to optimize bariatric surgery weight loss outcomes through identification of key mechanisms that can be targeted for intervention.

This will be the first prospective, longitudinal study to carefully examine the biobehavioral contributors to bariatric 
surgery weight loss trajectory. Most studies have been limited by short follow-up periods and small sample sizes. In contrast, the current sufficiently powered study includes postoperative follow-up assessments that capture multiple points before weight nadir (1, $6 \mathrm{mo})$, approaching weight nadir (12 mo), and after weight nadir when weight regain may occur $(18,24 \mathrm{mo})$ [3]. In addition, the majority of extant data involves either behavioral or biological etiologies rather than using an integrated approach. Limited existing evidence suggests bidirectional communication between the microbiome and brain function both in times of homeostasis and disease [21]. This communication seems to occur primarily through complex interactional effects in the hypothalamic-pituitary-adrenal axis and structures in the central nervous system that can affect cognition, mood, and emotion. There are currently very few data addressing the relationship between postbariatric surgery changes in the gut microbiota on behavior, and this prospective study will allow us to investigate the gut-brain axis through detailed and comprehensive assessments of the gut microbiota, psychopathology, eating behavior, food intake, and cognition.

This study will help answer whether the gut microbiome changes in response to diet and whether there are associations between the gut microbiome and problematic eating behaviors. The dramatic changes in nutrient intake that follow bariatric surgery are expected to alter the composition of the gut microbiome. Dietary intake over long periods of time plays a major role in shaping the composition and determining the diversity of the intestinal microbiota. The combined approach of $16 \mathrm{~S}$ and metagenomic shotgun sequencing will allow us to examine both taxonomic and functional changes to the microbiome that occur in response to surgery.

An increasing literature is exploring the likely bidirectional relationship between eating behavior and the gut microbiome. Among animal studies, yo-yo dieting may be as unhealthy as a steadily poor diet [53]. To date, there are limited data concerning the effects of anorexia nervosa on the microbiome [54-56], but the influence of binge eating on the intestinal microbiome remains an empirical question, and one that is being addressed in the present study.

The gut microbiome also appears to be sensitive to changes in physical activity. The majority of studies examining the effects of exercise on the intestinal microbiota, however, have been conducted in animal models and shows that exercise affects the diversity of the intestinal microbiome, especially the Bacteroidetes and Firmicutes phyla [57-60] and have not assessed this relationship in a bariatric sample

Furthermore, a growing number of studies have suggested that the gut microbiome plays a role in depression [61-63]. Inducing depression-like states in mice, has been found to result in measurable changes to the intestinal microbiome [64]. It appears that the gut microbiome also has the capacity to influence mood and anxiety symptoms [21]. Using 6 data collection time points spanning from before to 24 months after surgery, we will be able to characterize the temporal relationships between changes in the microbiota and changes in mood. Necessary future studies will be able to build upon data collected in this study, with the aim of exploring the gut-brain axis in greater detail among bariatric surgery patients. In particular, given the high rates of mood disorders present in the bariatric surgery population and the recurrence of mood symptoms that often occurs distal to surgery, it is particularly important to focus on clarifying mechanisms responsible for enduring or relapsing symptoms.

A growing number of studies also suggest that the gut microbiome is an important contributor to cognitive function. Though the mechanisms remain incompletely understood, gut-brain communication is mediated through multiple systems, including numerous cytokines [65]. Mice assigned to a high sucrose diet exhibit a greater reduction in Bacteroidetes and poorer performance on a measure of cognitive flexibility [66]. Similarly, mice receiving microbiome transplantation from those prescribed a highfat diet show poorer learning of new information and exploratory behavior than controls [67]. Few studies have directly examined the relationship between the gut microbiome and cognitive function in humans. An initial study in persons with cirrhosis compared those with and without hepatic encephalopathy and revealed a pathway from dysbiosis of microbiome to inflammatory processes to cognitive dysfunction in those with hepatic encephalopathy [68]. We have recently generated additional evidence of a positive association between cognition and the gut microbiome [69].

The present study will help elucidate the interaction between biological and behavioral variables and may be used as a framework from which to begin additional more detailed mechanistic assessments between specific significant associations with the gut microbiome and variables of interest after bariatric surgery.

This study will address limitations in previous, smaller investigations of the gut microbiome in bariatric surgery patients that may contribute to inconsistency among findings. Inconsistent results of prior studies may be attributable to several factors. There have been variations in study design, laboratory techniques used to characterize the microbiome, and sample sizes have generally been small. Additionally, limited attention has been given to controlling variables that may confound study results, such as dietary intake [20]. The consistent identification of changes in the microbiota of the gut after bariatric surgery, coupled with the inconsistent findings with respect to the downregulation or proliferation of specific bacterial species, supports the need for additional well-controlled research in this area. Each of these limitations will be strengthened by the present study proposal, which involves detailed collection of dietary intake information. 
The data captured in this study will be instrumental in moving toward precision medicine in bariatric surgical care. We will use these data to identify predictors of postsurgical weight loss outcome so that at-risk patients can be identified and preventative and therapeutic interventions can be implemented in a timely fashion. These data will also serve as the basis for designing subsequent psychotherapeutic and pharmacologic weight regain prevention and treatment options to help optimize bariatric surgery outcomes. Ultimately, these data may help to elucidate the mechanism(s) responsible for weight change and weight recidivism after bariatric surgery, which may help in the future development of nonsurgical, less invasive treatment options for severe obesity.

\section{Challenges and design considerations}

This study provides one approach to collecting data on the gut microbiome in the bariatric surgery population. This patient population presents numerous challenges to the collection of data on the gut microbiome, and requires a careful balance between limiting confounds and maintaining generalizability. Among the most significant design considerations was whether or not to exclude common disease states or surgical histories that are relatively common but may influence the gut microbiota, and importantly, may also change after surgery (e.g., diabetes, hypertension, history of cholecystectomy). Ultimately, we elected to include all disease states except those which are relatively rare among this patient population and expected to dramatically influence the gut microbiota (e.g., colon resection). Other major design considerations surrounded which medications to include as exclusionary criteria. Ultimately, the study team established a list of medications that would be expected to have a profound influence on the gut microbiota when taken routinely, and which may not be controllable analytically because of low base rates of use and possible changes from pre- to postsurgery in use, such as glucagon-like-peptide agonists, opioids, potent anticholinergics, and a few others with empirical or theoretic impact on the microbiome. Acid-reducing medications, despite their known impact on the microbiota, were permitted because of their pervasive use among this population. An additional major variable we elected to control for by excluding participants or carefully timing baseline data collection was presurgery weight loss medications and dietary prescriptions (i.e., liquid diet before surgery), which we expected to have a major impact on weight trajectory and the gut microbiota.

\section{Conclusions}

Overall, this prospective, multicenter study will provide data from a large sample of bariatric surgery patients who are followed for 2 years postoperatively. Data will include comprehensive and validated assessments of behavior, along with assessment of the gut microbiome using contemporary shotgun sequencing methodology. We will be able to compare these relationships among patients who undergo RYGB with those who undergo SG. These data will provide an important framework from which to develop subsequent more targeted protocols exploring specific aspects of the gut microbiome and its mechanistic influence on bariatric surgery outcomes. Although the focus of this study is on postbariatric surgery weight outcomes, data concerning obesity-associated co-morbidity resolution and reoccurrence are also being collected. Increasingly, data suggest that the gut microbiome is an important regulator of obesity-associated co-morbidities, such as diabetes and cardiovascular disease. Data collected through this study may pave the way to more tailored investigations exploring the mechanisms through which the gut microbiota impacts various disease states and psychopathology.

\section{Disclosures}

The authors have no commercial associations that might be a conflict of interest in relation to this article.

\section{References}

[1] Buchwald H, Avidor Y, Braunwald E, et al. Bariatric surgery: a systematic review and meta-analysis. JAMA 2004;292(14):1724-37.

[2] ASMBS. Estimate of Bariatric Surgery Numbers, 2011-2018 [cited 2020 Jun 7]. Available from: https://asmbs.org/resources/estimateof-bariatric-surgery-numbers.

[3] Courcoulas AP, Christian NJ, Belle SH, et al. Weight change and health outcomes at 3 years after bariatric surgery among individuals with severe obesity. JAMA 2013;310(22):2416-25.

[4] Karmali S, Brar B, Shi X, Sharma AM, de Gara C, Birch DW. Weight recidivism post-bariatric surgery: a systematic review. Obes Surg 2013;23(11):1922-33.

[5] Kofman MD, Lent MR, Swencionis C. Maladaptive eating patterns, quality of life, and weight outcomes following gastric bypass: results of an internet survey. Obesity 2010;18(10):1938-43.

[6] Diamantis T, Apostolou KG, Alexandrou A, Griniatsos J, Felekouras E, Tsigris C. Review of long-term weight loss results after laparoscopic sleeve gastrectomy. Surg Obes Relat Dis 2014;10(1):177-83.

[7] Meany G, Conceicao E, Mitchell JE. Binge eating, binge eating disorder and loss of control eating: effects on weight outcomes after bariatric surgery. Eur Eat Disord Rev 2014;22(2):87-91.

[8] White MA, Kalarchian MA, Masheb RM, Marcus MD, Grilo CM. Loss of control over eating predicts outcomes in bariatric surgery patients: a prospective, 24- month follow-up study. J Clin Psychiatry 2010;71(2): 175-84.

[9] Sheets CS, Peat CM, Berg KC, et al. Post-operative psychosocial predictors of outcome in bariatric surgery. Obes Surg 2015;25(2):330-45.

[10] Colles SL, Dixon JB, O’Brien PE. Grazing and loss of control related to eating: two high-risk factors following bariatric surgery. Obesity 2008;16(3):615-22.

[11] Faria SL, Kelly E, Faria OP. Energy expenditure and weight regain in patients submitted to Roux-en-Y gastric bypass. Obes Surg 2009;19(7):856-9.

[12] Lanyon RI, Maxwell BM, Kraft AJ. Prediction of long-term outcome after gastric bypass surgery. Obes Surg 2009;19(4):439-45. 
[13] White MA, Kalarchian MA, Levine MD, Masheb RM, Marcus MD, Grilo CM. Prognostic significance of depressive symptoms on weight loss and psychosocial outcomes following gastric bypass surgery: a prospective 24-month follow-up study. Obes Surg 2015;25(10):1909-16.

[14] Livhits M, Mercado C, Yermilov I, et al. Exercise following bariatric surgery: a systematic review. Obes Surg 2010;20(5):657-65.

[15] Mundi MS, Lorentz PA, Swain J, Grothe K, Collazo-Clavell M. Moderate physical activity as predictor of weight loss after bariatric surgery. Obes Surg 2013;23(10):1645-9.

[16] Bond DS, Phelan S, Wolfe LG, et al. Becoming physically active after bariatric surgery is associated with improved weight loss and healthrelated quality of life. Obesity 2009;17(1):78-83.

[17] Galioto R, Gunstad J, Heinberg LJ, Spitznagel MB. Adherence and weight loss outcomes in bariatric surgery: does cognitive function play a role? Obes Surg 2013;23(10):1703-10.

[18] Sweeney TE, Morton JM. Metabolic surgery: action via hormonal milieu changes, changes in bile acids or gut microbiota? A summary of the literature. Best Pract Res Clin Gastroenterol 2014;28(4):727-40.

[19] Husen Z, DiBaise JK, Zuccolo A, et al. Human gut microbiota in obesity and after gastric bypass. Proc Natl Acad Sci U S A 2009; 106(7):2365-70.

[20] Cani PD. Metabolism in 2013: The gut microbiota manages host metabolism. Nat Rev Endocrinol 2014;10(2):74-6.

[21] Mayer EA. Gut feelings: the emerging biology of gut-brain communication. Nat Rev Neurosci 2011;12(8):453-66.

[22] First MB, Spitzer RL, Gibbon M, Williams JB. Structured clinical interview for DSM-IV-TR axis I disorders, research version, patient edition. $(S C I D-I / P)$. Research Version ed. New York, NY: Biometrics Research, New York State Psychiatric Institue November; 2002.

[23] Moshfegh AJ, Rhodes DG, Baer DJ, et al. The US Department of Agriculture Automated Multiple-Pass Method reduces bias in the collection of energy intakes. Am J Clin Nutr 2008;88(2):324-32.

[24] Kipnis V, Subar AF, Midthune D, et al. Structure of dietary measurement error: results of the OPEN biomarker study. Am J Epidemiol 2003;158(1):14-21. discussion 22-6.

[25] Beck AT, Steer RA. Manual for revised Beck Depression Inventory, New York, NY: Psychology Corporation; 1987.

[26] Beck AT, Steer RA. Psychometric properties of the Beck Depression Inventory - 25 years of evaluation. Clin Psychol Rev 1988;8(1):77100.

[27] Watson D, Clark LA. Negative affectivity: the disposition to experience aversive emotional states. Psychol Bull 1984;96:465-90.

[28] Fairburn CG, Cooper Z, Wilson G. The eating disorder examination. In: Fairburn CG, editor. Binge eating: nature, assessment and treatment. 12 ed., New York, NY: Guilford Press; 1993. p. 317-60.

[29] Welch G, Wesolowski C, Piepul B, Kuhn J, Romanelli J, Garb J. Physical activity predicts weight loss following gastric bypass surgery: findings from a support group survey. Obes Surg 2008;18(5):517-24.

[30] Aadland E, Ylvisåker E. Reliability of the actigraph GT3 X+ accelerometer in adults under free-living conditions. PLoS One $2015 ; 10(8): 1-10$.

[31] Staudenmayer J, He S, Hickey A, Sasaki J, Freedson P. Methods to estimate aspects of physical activity and sedentary behavior from highfrequency wrist accelerometer measurements. J Appl Physiol 2015;119(4):396-403.

[32] King WC, Chen J-Y, Bond DS, et al. Objective assessment of changes in physical activity and sedentary behavior: pre- through 3 years postbariatric surgery. Obesity 2015;23(6):1143-50.

[33] Kim Y, Welk GJ, Braun SI, Kang M. Extracting objective estimates of sedentary behavior from accelerometer data: measurement considerations for surveillance and research applications. PLoS One 2015;10(2):1-15.

[34] Cognition measures [homepage on the Internet]. Evanston: Northwestern Univserity; c2020 [cited 2016 Apr 12]. Availabe from:
http://www.nihtoolbox.org/WhatAndWhy/Cognition/Pages/default. aspx.

[35] Spitznagel MB, Alosco M, Strain G, et al. Cognitive function predicts 24-month weight loss success after bariatric surgery. Surg Obes Relat Dis 2013;9(5):765-70.

[36] Spitznagel MB, Alosco M, Galioto R, et al. The role of cognitive function in postoperative weight loss outcomes: $36-$ month follow-up. Obes Surg 2014;24(7):1078-84.

[37] Carroll IM, Ringel-Kulka T, Keku TO, et al. Molecular analysis of the luminal- and mucosal-associated intestinal microbiota in diarrheapredominant irritable bowel syndrome. Am J Physiol Gastrointest Liver Physiol 2011;64(5). G799-807.

[38] Arthur JC, Perez-Chanona E, Mühlbauer M, et al. Intestinal inflammation targets cancer-inducing activity of the microbiota. Science 2012;338(6103):120-3.

[39] Callahan BJ, McMurdie PJ, Rosen MJ, Han AW, Johnson AJ, Holmes SP. DADA2: high-resolution sample inference from Illumina amplicon data. Nat Methods 2016;13(7):581-3.

[40] McCafferty J, Mühlbauer M, Gharaibeh RZ, et al. Stochastic changes over time and not founder effects drive cage effects in microbial community assembly in a mouse model. ISME J 2013;7:2116-25.

[41] Caporaso JG, Kuczynski J, Stombaugh J, et al. QIIME allows analysis of high-throughput community sequencing data. Nat Methods 2010;7(5):335-6.

[42] Edgar RC. MUSCLE: a multiple sequence alignment method with reduced time and space complexity. BMC Bioinformatics 2004;5:113-9.

[43] Edgar RC. Search and clustering orders of magnitude faster than BLAST. Bioinformatics 2010;26(19):2460-1.

[44] Wang Q, Garrity GM, Tiedje JM, Cole JR. Naive Bayesian classifier for rapid assignment of rRNA sequences into the new bacterial taxonomy. Appl Environ Microbiol 2007;73(16):5261-7.

[45] Lozupone C, Knight R. UniFrac: a new phylogenetic method for comparing microbial communities. Appl Environ Microbiol 2005;71(12):8228-35.

[46] Wood DE, Salzberg SL. Kraken: ultrafast metagenomic sequence classification using exact alignments. Genome Biol 2014;15. R46-12.

[47] Franzosa EA, McIver LJ, Rahnavard G, et al. Species-level functional profiling of metagenomes and metatranscriptomes. Nat Meth 2018;15:962-8.

[48] Kalbfleisch JD, Prentice RL. The statistical analysis of failure time data, New York, NY: John Wiley \& Sons; 1980.

[49] Singer JD, Willett JB. Applied longitudinal data analysis: modeling change and event occurrence, New York, NY: Oxford University Press; 2003.

[50] Preacher KJ, Zyphur MJ, Zhang Z. A general multilevel SEM framework for assessing multilevel mediation. Psychol Methods 2010;15(3):209-33.

[51] Mplus users' guide. 6th ed., Los Angeles, CA: Muthen \& Muthen; 1998-2011.

[52] Fritz MS, MacKinnon DP. Required sample size to detect the mediated effect. Psychol Sci 2007;18(3):233-9.

[53] Kaakoush NO, Martire SI, Raipuria M, et al. Alternating or continuous exposure to cafeteria diet leads to similar shifts in gut microbiota compared to chow diet. Mol Nutr Food Res 2016;61(1):1-17.

[54] Kleiman SC, Watson HJ, Bulik-Sullivan EC, et al. The intestinal microbiota in acute anorexia nervosa and during renourishment: relationship to depression, anxiety, and eating disorder psychopathology. Psychosom Med 2015;77(9):969-81.

[55] Kleiman SC, Carroll IM, Tarantino LM, Bulik CM. Gut feelings: a role for the intestinal microbiota in anorexia nervosa? Int J Eat Disord 2015;48(5):449-51.

[56] Carr J, Kleiman SC, Bulik CM, Bulik-Sullivan EC, Carroll IM. Can attention to the intestinal microbiota improve understanding and 
treatment of anorexia nervosa? Expert Rev Gastroenterol Hepatol 2016;10(5):565-9.

[57] Choi JJ, Eum SY, Rampersaud E, Daunert S, Abreu MT, Toborek M. Exercise attenuates PCB-induced changes in the mouse gut microbiome. Environ Health Perspect 2013;121(6):725-30.

[58] Evans CC, LePard KJ, Kwak JW, et al. Exercise prevents weight gain and alters the gut microbiota in a mouse model of high fat diet-induced obesity. PLoS One 2014;9(3):e92193.

[59] Lambert BS, Shimkus KL, Fluckey JD, et al. Anabolic responses to acute and chronic resistance exercise are enhanced when combined with aquatic treadmill exercise. Am J Physiol Endocrinol Metab 2015;308(3). E192-200.

[60] Petriz BA, Castro AP, Almeida JA, et al. Exercise induction of gut microbiota modifications in obese, non-obese and hypertensive rats. BMC Genomics 2014:15:511.

[61] Collins SM, Kassam Z, Bercik P. The adoptive transfer of behavioral phenotype via the intestinal microbiota: experimental evidence and clinical implications. Curr Opin Microbiol 2013;16(3):240-5.

[62] Liang S, Wang T, Hu X, et al. Administration of Lactobacillus helveticus NS8 improves behavioral, cognitive, and biochemical aberrations caused by chronic restraint stress. Neuroscience 2015;310:561-77.
[63] Dinan TG, Cryan JF. Melancholic microbes: a link between gut microbiota and depression? J Neurogastroenterol Motil 2013;25(9):713-9.

[64] Park AJ, Collins J, Blennerhassett PA, et al. Altered colonic function and microbiota profile in a mouse model of chronic depression. J Neurogastroenterol Motil 2013;25(9). 733-e575.

[65] Saulnier DM, Ringel Y, Heyman MB, et al. The intestinal microbiome, probiotics and prebiotics in neurogastroenterology. Gut Microbes 2013;4(1):17-27.

[66] Magnusson KR, Hauck L, Jeffrey BM, et al. Relationships between diet-related changes in the gut microbiome and cognitive flexibility. Neuroscience 2015;300:128-40.

[67] Bruce-Keller AJ, Salbaum JM, Luo M, et al. Obese-type gut microbiota induce neurobehavioral changes in the absence of obesity. Biol Psychiatry 2015;77(7):607-15.

[68] Bajaj JS, Hylemon PB, Ridlon JM, et al. Colonic mucosal microbiome differs from stool microbiome in cirrhosis and hepatic encephalopathy and is linked to cognition and inflammation. Am J Physiol Gastrointest Liver Physiol 2012;303(6):G675-85.

[69] Manderino L, Carroll I, Azcarate-Peril MA, et al. Preliminary evidence for an association between the composition of the gut microbiome and cognitive function in neurologically healthy older adults. J Int Neuropsychol Soc 2017;23(8):700-5. 